\title{
Effect of channel radius on the molecular communication based on fluorescent nanoparticles
}

\author{
Federico Calì ${ }^{1}$, Luca Fichera ${ }^{1}$ and Nunzio Tuccitto ${ }^{1,2, *}$
}

1 Department of Chemical Sciences, Università degli Studi di Catania, Viale A. Doria 6, 95125 Catania, Italy; federico.cali@phd.unict.it (F.C.); luca.fichera@phd.unict.it (L.F.)

2 Consorzio per lo Sviluppo dei Sistemi a Grande Interfase, CSGI, Viale A. Doria 6, 95125 Catania, Italy

* Correspondence: nunzio.tuccitto@unict.it (N.T.)

\begin{abstract}
The effect of the communication channel size on the transport and subsequent detection of chemical messengers is investigated on millimetric and micrometric channels. The transport of the information carriers, being characterized by an advective and a diffusive contribution, was simulated by varying the flow velocity and the diffusion coefficient. Then, to evaluate the information quality, the Intersymbol Interference (ISI) between two consecutive signals at a specific release delay was estimated. This allowed us to verify that operating under micrometric channel conditions has a larger flow velocity range to obtain completely separated successive signals and smaller release delays can be used between signals. The theoretical results were confirmed by developing a prototype molecular communication platform operating under microfluidic conditions, which enables communication through fluorescent nanoparticles, namely Carbon Quantum Dots (CQDs).
\end{abstract}

Keywords: molecular communication; microfluidics; intersymbol interference; carbon quantum dots; flow velocity; diffusion coefficient

\section{Introduction}

Nowadays, one of the technological fields in which there is the greatest rush of scientific research is communications. Once we have made society hyper-connected thanks to the advent of 5G, a branch of communications research is aspiring to solve a very different problem, namely communication in environments where information cannot be transferred by electromagnetic waves. There are essentially two reasons why communication cannot take place by means of electromagnetic waves: the first is when there are barriers or obstacles that physically impede the propagation of the wave ${ }^{1}$. The second concerns the possible harmful consequences of the waves propagating in a given environment, for example in close contact with or buried within biological systems ${ }^{2}$. An alternative approach to transporting information by electromagnetic waves ${ }^{3}$ was theorized more than a decade ago and is based on the use of chemical information carriers (ICs), for example molecules or particles ${ }^{4}$. This artificial chemical communication is known in the scientific community as Molecular Communication $(\mathrm{MoCo})^{5-7}$. Molecular Communication, although conceived in recent times, refers to and imitates chemical and biological mechanisms that have always existed and that nature has perfected to allow communication between living species of any order of complexity, but also to regulate biological cycles and allow the development of life itself ${ }^{8,9}$. It is, in fact, a bio-inspired approach. In order to reproduce this mechanism, we need a platform with three main components, as in traditional communication systems: a transmitter, a communication channel and a receiver ${ }^{10}$. The transmitter is able to encode the message and release a small number of information carriers; these will propagate through the communication channel which, in most cases, consists of a liquid or gaseous medium. At the end of their journey within the communication channel, the ICs will encounter the receiver, which allows the detection and subsequent decoding of the message. A representative scheme of this mechanism can be seen in Figure 1. 


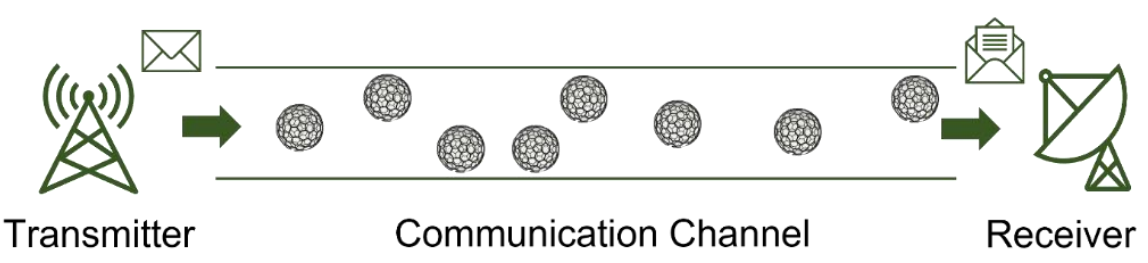

Figure 1. Schematic representation of an artificial molecular communication system considering spherical nanoparticles as information carriers.

Depending on the distances covered by the messengers from the transmitter to the receiver, but above all on the intrinsic size of the communication channel, molecular communication can be classified into macro - molecular communication (Macro - MoCo) ${ }^{11}$, micro - molecular communication (Micro - MoCo) ${ }^{12}$, and nano - molecular communication (Nano - MoCo $)^{13}$. In this paper we focused on the first two conditions. Based on the different channel size, the contexts in which molecular communication would find an application also change ${ }^{14}$. Environments where Macro - MoCo can be applied are underground or underwater, which are strategic for the well-being of cities or for the improvement of agricultural systems. Practical examples are sewerage and water distribution systems in cities, or channels for sub-irrigation, sub-fertilization, and sub-treatment in agriculture. But Macro - MoCo could also benefit all those companies and industries characterized by the presence of extensive and complex steel structures that prevent the propagation of waves and therefore make wireless communication almost impossible ${ }^{15}$. Micro MoCo is increasingly used in biological contexts and in the medical study of new and sophisticated methods of monitoring vital parameters for the treatment or prevention of certain diseases ${ }^{16}$. From a more general point of view, it is a type of communication that would take place by means of the micrometer-sized blood vessels and capillaries in animals. For medical purposes, the optimum would be for two or more bio-implanted devices to communicate ${ }^{17}$, at least one of which allows the reading of the variation of a strategic parameter and the release of information carriers. The latter, through the biological fluids, will go to the second device, which allows the decoding of the message and the subsequent dosage of the drug useful in preventing or treating the disease ${ }^{18}$. There are also other biological contexts in which more or less complex vascular systems can be exploited, in addition to the human one. This is the example of plants, which are also characterized by channels that allow the transport of vital lymph, and which are classified into xylem channels and phloem channels. The xylem channels allow the transport of water and minerals, while the phloem channels transport the products of the photosynthesis process that takes place in the leaves ${ }^{19,20}$. Then there is a type of molecular communication that happens at the nanoscale typical of intracellular biochemical phenomena ${ }^{21}$.

Here we propose a study, first theoretical and then experimental, which aims to highlight the differences and possible limitations of transporting information carriers by an aqueous fluid in Macro-MoCo occurring trough communication channels having micrometric or millimetric diameters. Different channel diameters mean transporting the ICs under different fluidic phenomena. What we have investigated is the presence or absence of a peculiar fluid dynamic phenomenon well-known in the literature as Taylor Diffusion. This mechanism can be observed whenever a fluid is transported along a channel of cylindrical section under Poiseuille laminar conditions. ${ }^{22}$ Poiseuille's Law is given by equation 1 ,

$$
v(r)=\frac{\Delta P}{4 \eta L}\left(R^{2}-r^{2}\right)
$$

where $\mathrm{v}$ is the velocity, $\Delta \mathrm{P}$ is the pressure difference imposed between the two ends, $\eta$ is the fluid viscosity, and $L$ is the channel length. The equation implies that the velocity of the fluid decreases radially from a maximum $\mathrm{V}_{\max }$ at the center of the cylinder $(\mathrm{r}=0)$ to a 
minimum $\mathrm{V}_{\min }$ at the walls of the cylinder $(\mathrm{r}=\mathrm{R})$. This condition gives the axial spreading of the fluid, namely advection. Under advection, a plug of solute transported by the flow will assume a typical arrow-shaped profile due to the laminar flow. Regions of concentration gradient will develop at the front and back ends of the solute plug, causing a radial diffusion. This radial diffusion is called Taylor Diffusion, named after the first scientist to study the phenomenon ${ }^{23}$, of which the role is to limit the axial spreading due to advection, slowing down the anterior end and speeding up the posterior end of the solute. The total effect of the combination of the two contributes, advection and radial diffusion respectively, is to develop a strongly symmetrical concentration distribution of the solute during its journey ${ }^{24}$. This results in an expected signal to the receiver placed at a distance $L$ from the transmitter characterized by a Gaussian distribution. Thus, the solute concentration at different values of $t$ is approximately given by equation 2 ,

$$
C \propto C_{0} e^{-\frac{\left(t-t_{0}\right)^{2}}{2 \sigma^{2}}},
$$

where $\mathrm{C}_{0}$ is the initial solute concentration and $\sigma^{2}$ is the variance.

The greater the diffusion coefficient of the transported substance, the greater the effect of Taylor Diffusion. The diffusion coefficient can be estimated according to the StokesEinstein equation,

$$
\mathrm{D}=\frac{\mathrm{k}_{\mathrm{B}} \mathrm{T}}{6 \pi \eta \mathrm{r}_{\mathrm{h}}}
$$

where $\mathrm{k}_{\mathrm{B}}$ is the Boltzmann constant, $\mathrm{T}$ is the temperature, $\mathrm{rn}$ is the hydrodynamic radius of the solute, in our case the information carriers, and $\eta$ is the fluid viscosity.

For the first time, we explored two different conditions regarding the transport of the ICs in molecular communication: the first one, occurring in millimetric channel (mChannel), in which cannot be observed Taylor regime, and the second one, in micrometric channel ( $\mu$ Channel), affected by Taylor regime. To analyze the two cases, one parameter we have assessed is the Intersymbol Interference (ISI), which is a key variable in optimizing molecular communication 25,26 .

First of all, we numerically solve simulations concerning the transport of the ICs. Simulations allow us to study the phenomenon from a theoretical point of view, but also to find the best conditions for our purposes by avoiding a large number of experiments. Knowing that the decoding of the message by the receiver is based on the counting of the information carriers arriving at the receiver itself, and therefore on the detection of their concentration ${ }^{27}$, the transport of the ICs is studied by numerically solving the Advection Diffusion Equation (ADE),

$$
\frac{\partial C}{\partial t}=\nabla \cdot\left(D_{I C} \nabla C\right)-\nabla \cdot(v C)
$$

where $\mathrm{C}$ is the concentration value of the information carriers, Dic is their diffusion coefficient and $\mathrm{v}$ is the term representing the velocity field along the transport channel. For our purposes, we simulated the transport of the messengers considering different velocity flow values, diffusion coefficients and channel diameters. To evaluate ISI effects, we simulated the sending of two consecutive bits with a specific release delay between one and the other. Then, we assembled a prototypal platform, and we undertook an experimental campaign to validate the theoretical results. To carry out a typical MoCo experiment we had to choose information carriers that had specific requirements, such as solubility in aqueous media, easy synthesis, low or no toxicity in biological matrices and a chemical or physical property that would allow them to be detected by the receiver. We employed fluorescent nanoparticles as information carriers, the well-known Carbon Quantum Dots (CQDs) ${ }^{28-30}$. CQDs meet all the above requirements to be good information carriers. Their intense and easily modulable fluorescence ${ }^{31}$ is the main property that we exploit to make them selectively detectable by the receiver. 


\section{Materials and Methods}

Simulations have been performed by the authors using Python programming language and its useful numerical algebra library named NUMPY and the finite volume partial differential equations solver named FiPy. Graph resulting from simulations were prepared using the Matplotlib library.

The Carbon Quantum Dots used in this work were prepared from citric acid (Merck, Italy), using the pyrolysis method. A quantity of about 10 grams of citric acid is weighed and placed inside a beaker on a heating plate at a temperature between 200 and $250{ }^{\circ} \mathrm{C}$. This pyrolysis process produces a dark brown caramel after 10 to 15 minutes. This is cooled to room temperature and then a $0.2 \mathrm{M} \mathrm{NaOH}$ solution (Merck, Italy) is added to it until a neutral $\mathrm{pH}$ is reached. The resulting suspension is subjected to membrane dialysis (Membra-Cel MC18 with a molecular weight cut-off of 11,000 Daltons) to remove all water-soluble pyrolysis residues and by-products. Dialysis is continued, periodically changing the water, until non-fluorescent dialysis water is obtained (typically after 2 days). Finally, a cryo-centrifugation step is carried out for 1 hour at $6000 \mathrm{rpm}$ and $3^{\circ} \mathrm{C}$. The supernatant is recovered and subjected to subsequent characterization and MoCo experiments. The typical concentration of the 'as prepared' solution is around $2 \mathrm{mg} / \mathrm{ml}$. For characterization of CDs and related instrumentation see article ${ }^{32}$.

The prototype platform we assembled to conduct molecular communication experiments consists of three main components, namely a transmitter, a communication channel and a receiver. The transmitter consists of an infusion pump (IVAC P3000), a six-way injection valve to release the nanoparticles coming from a selection valve enables to sample from ten different wells (both valves from Rheodyne, IDEX Health \& Science). The schematic representation of the coordinated work of the two valves during the loading and releasing processes is available in figure 2.

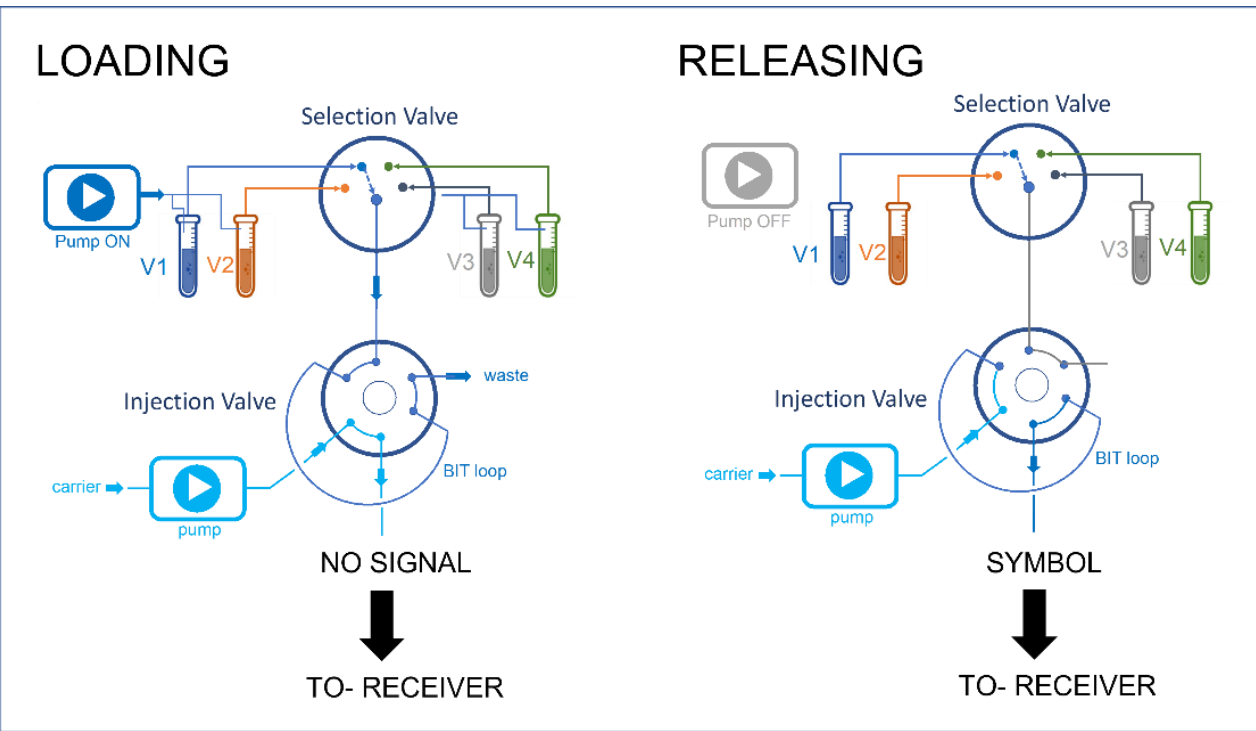

Figure 2. Schematic representation of the loading and realising steps co-ordinately governed by selection and injection valves.

A microfluidic communication channel was used, having diameter of $750 \mu \mathrm{m}$ (PTFE tube ID 1/32" OD 1/16"), and length of $30 \mathrm{~cm}$. We used a fluorescence detector (Shimadzu, rf535) as receiver, setting it at $365 \mathrm{~nm}$ excitation and $450 \mathrm{~nm}$ emission. Carrier solutions and CQDs suspensions having different viscosity were prepared by mixing water (ultrapure filtered, Millipore Merck, Italy) and glycerol (Merck, Italy) until the desired viscosity was achieved. The viscosity was measured with a viscometer tube (Cannon-Fenske, ASTM D446). 


\section{Results}

The modulation method we considered to realize simulations and experiments is the "On - Off Keying" modulation (OOK), which is a particular case of the "Concentration Shift Keying" (CSK) ${ }^{33}$. For the CSK, information can be encoded according to the transmitter concentration of information carriers. With this modulation is possible to transfer information in binary code, encoding the signal 1-bit by releasing ICs with a concentration above a predetermined threshold and 0-bit if lower. Usually, concentration threshold can be defined as the minimum limit of detection (LOD) of the detector used as receiver, or just above the limit of interference-generated noise. A simpler case is represented by $\mathrm{OOK}^{34}$, where there is not any concentration threshold, thus 1-bit is given by a ICs concentration sent from the transmitter different from zero ( $\left.\mathrm{CICs}_{\mathrm{I}} \neq 0\right)$ and 0 -bit is achieved when the transmitter does not release ICs $(\mathrm{CICS}=0)$.
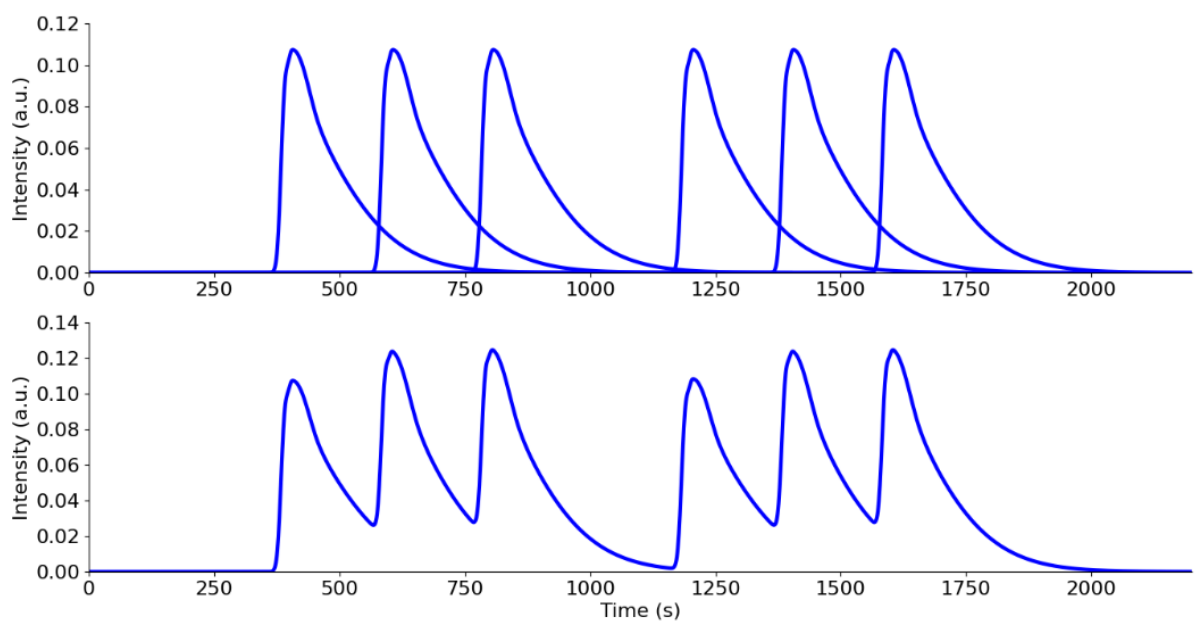

Figure 3. Binary encoded sequence of 8 consecutive bits representative of the letter " $w$ " in ASCII language. Signals as they should be without ISI (upper graph), signals as they are with ISI (lower graph).

Considering OOK modulation method, ISI occurs when two neighboring signals in sequence present a certain degree of overlap, causing an error in reading the symbol attributed to those signals if the aspect ratio is lower than the detector sensitivity. To better understanding the phenomenon, we report an illustrative example in figure 3 . It represents a simulated sequence of 8 consecutive bits (solving equation 4), binary encoded, sent with a delay of 200 seconds from each other. The sequence is 01110111, where 1-bit is given by the presence of the signal (in realistic case, this means non - zero concentration of the information carries read by the receiver) and 0-bit is given by the absence of the signal (concentration of the information carries equal to zero). Sequence 01110111 gives " $w$ " letter in ASCII language. It is very clear the difference between the upper sequence, evidencing what the signals should look like without ISI, and the bottom sequence where ISI occurs and can be observed the overlap between signals. 
Figure 4 shows simulation results for the transport of a substance plug in a mChannel having radius $1.2 \mathrm{~mm}$ and length $30 \mathrm{~cm}$. We investigated how change the signal properties, i.e. shape and intensity, modifying velocity flow (advective term) and keeping constant diffusion coefficient (diffusive term), and vice versa.

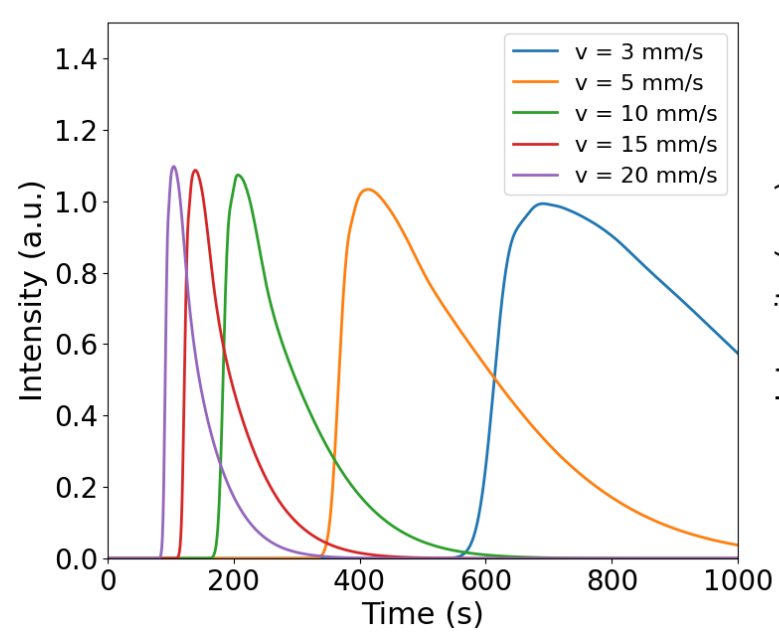

(a)

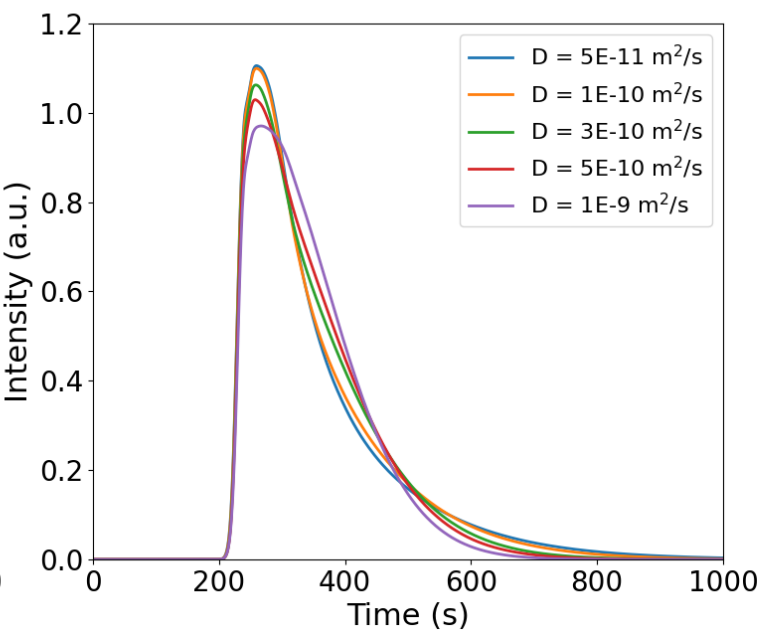

(b)

Figure 4. Simulated signals obtained in a channel having radius $1.2 \mathrm{~mm}$ and length $30 \mathrm{~cm}$; (a) colours are associated with different values of flow velocity, while a constant diffusion coefficient of $3 \mathrm{E}-10 \mathrm{~m} / \mathrm{s}$ is used; (b) colours are associated with different values of diffusion coefficient, while a constant flow velocity of $8 \mathrm{~mm} / \mathrm{s}$ is used.

Simulated signals represented in figure 4 a were obtained by changing the flow velocity and keeping constant the diffusion coefficient having a value of $3 \mathrm{E}-10 \mathrm{~m}^{2} / \mathrm{s}$. This value was chosen based on information nanoparticles with a typical size of about $2 \mathrm{~nm}^{35}$. It can be observed that none of the signals in question have a Gaussian distribution but are all curves with distorted symmetry. This is caused by the parabolic flow velocity profile, whereby what is in the center of the channel travels faster and reaches the receiver sooner, while what is near the walls is transported more slowly, causing a long tail in the signal (tailing effect). Transport is a non - homogeneous process. As the flow velocity decreases, it is noticeable how the dispersion increases, manifesting itself in increasingly less intense and sparse signals. On the other hand, an increase in flow velocity allows the substance to stay more compact during its journey from transmitter to receiver and the signal will be more intense. Figure $4 \mathrm{~b}$ shows that significant changes in the diffusion coefficient have very little effect on the shape and intensity of the signals. Considering an intermediate value of flow velocity between those considered in figure 4a equal to $8 \mathrm{~mm} / \mathrm{s}$ and varying $\mathrm{D}$ by almost 2 orders of magnitude always results in highly distorted Gaussian distributions. With mChannel sizes, while analyzing operating ranges of flow velocity and diffusion coefficient typical of molecular communication experiments, non-Gaussian signals are obtained. 

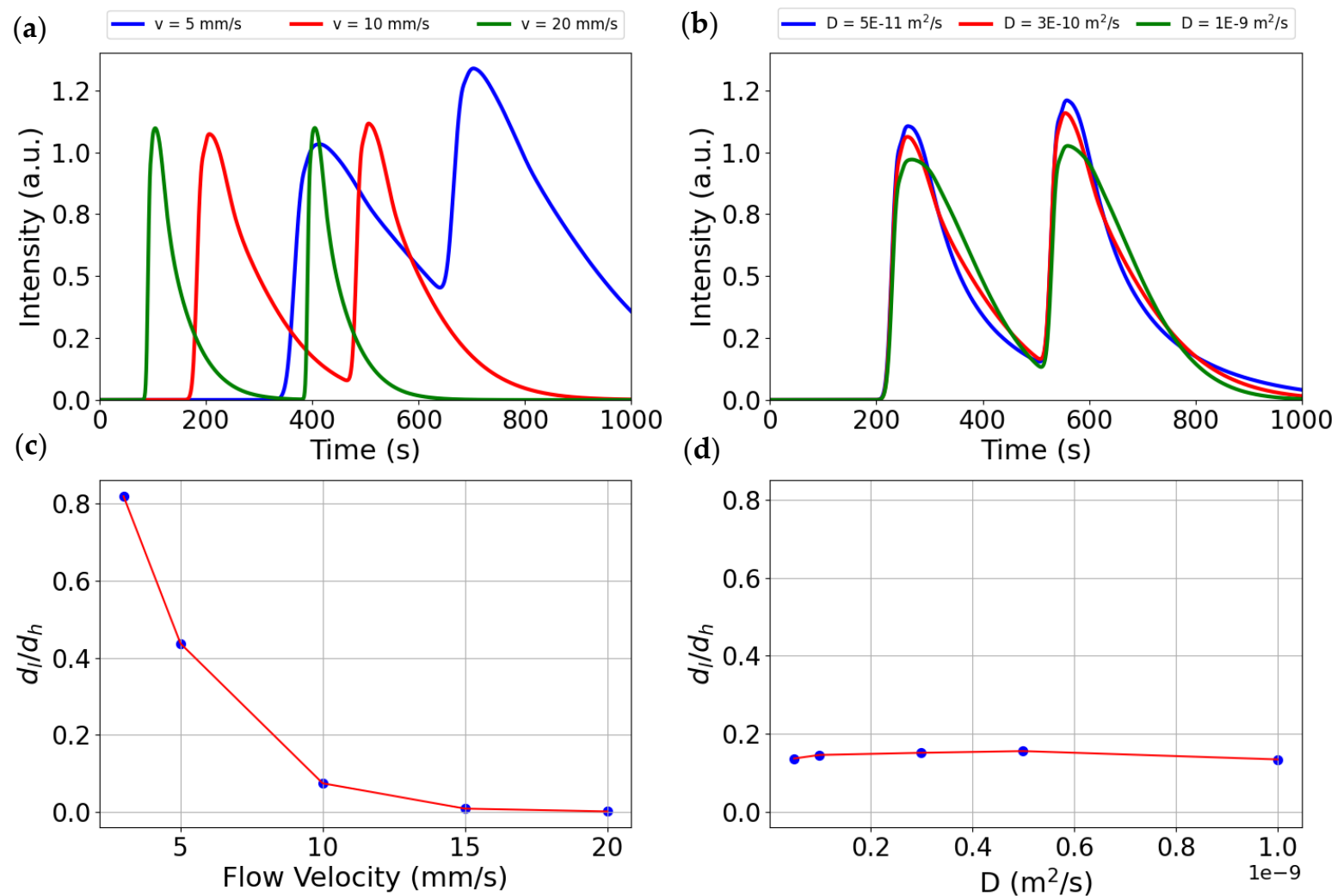

Figure 5. $(a, b)$ Simulated signals representing two consecutive bits with a fixed delay between them of 300 seconds, colours are associated with different values of flow velocity and diffusion coefficient, respectively; $(\mathrm{c}, \mathrm{d})$ development of the $\mathrm{d}_{\mathrm{l}} / \mathrm{dh}_{\mathrm{h}}$ ratio as a function of flow velocity and diffusion coefficient, respectively.

Figures $5 a$ and $5 b$ show how the effect of ISI varies in mChannel as the flow velocity and diffusion coefficient change, respectively. For this study, a fixed delay between two consecutive bits of 300 seconds was chosen. Considering only three different values of flow velocity and diffusion coefficient to make the graphs clearer, it can be easily observed that flow velocity strongly influences ISI and that specifically as the flow velocity decreases, the signals interference increases; on the other hand, variations of even two orders of magnitude in the diffusion coefficient do not influence ISI. To express this result quantitatively, we plotted the trend of the $\mathrm{dl} / \mathrm{dh}$ ratio against both the flow rate and the diffusion coefficient, where $\mathrm{dl}$ indicates the intensity of the "valley" between two consecutive bits and dh the maximum intensity of the first bit. The two borderline cases of this relationship are $d_{l} / d_{h}=0$ and $d_{l} / d_{h}=2$, the first one means that the two consecutive bits are perfectly separated and the second one means that two consecutive bits are totally overlapped so as to observe only one, with twice the intensity of the single bit. Figure 5 c shows that this ratio covers a relatively wide range, from about 0.8 to 0 as the flow velocity increases; instead, figure $5 \mathrm{~d}$ shows a much narrower range of the $\mathrm{d}_{1} / \mathrm{d}_{\mathrm{h}}$ ratio than the first, depicting values between approximately 0.135 and 0.155 . This is further confirmation of what has been described by figures $5 \mathrm{a}$ and $5 \mathrm{~b}$. Similar results are obtained with larger channel radii, even with more pronounced effects. 


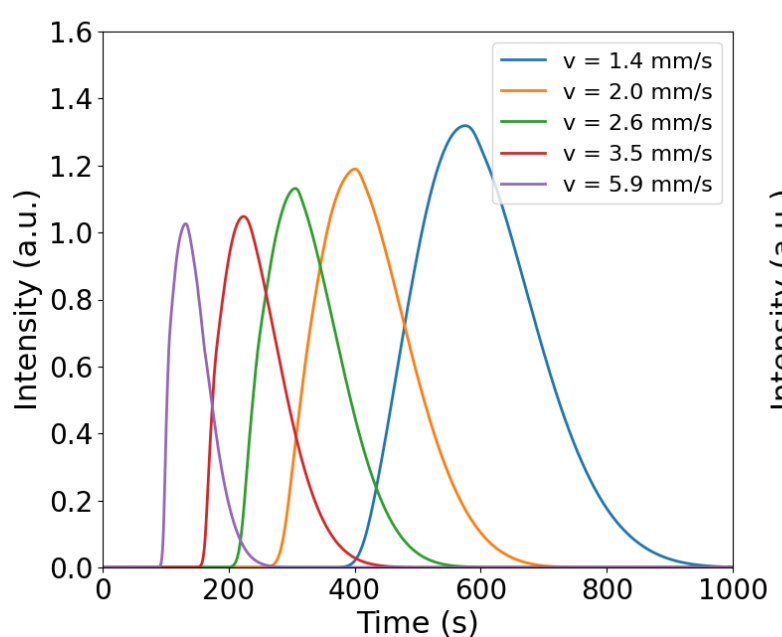

(a)

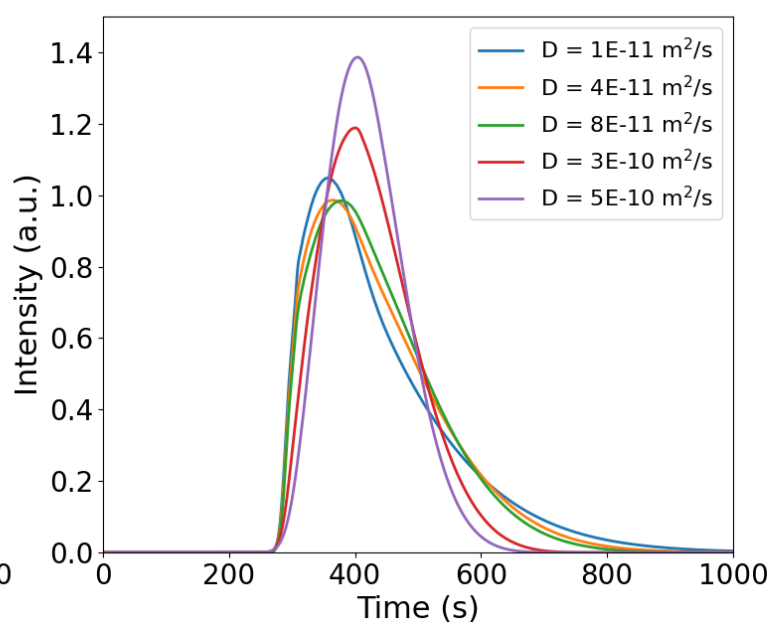

(b)

Figure 6. Simulated signals obtained in a channel having radius $0.375 \mathrm{~mm}$ and length $30 \mathrm{~cm}$; (a) colours are associated with different values of flow velocity, while a constant diffusion coefficient of $3 \mathrm{E}-10 \mathrm{~m}^{2} / \mathrm{s}$ is used; (b) colours are associated with different values of diffusion coefficient, while a constant flow velocity of $2 \mathrm{~mm} / \mathrm{s}$ is used.

We investigated $\mu$ Channel the communication occurring in pipe having internal diameter (ID) of $1 / 32$ " (about $0.375 \mathrm{~mm}$ radius) mostly used in common microfluidic experiments. Figure 7 a shows a trend of the simulated signals at varying flow velocities which is quite different from the mChannel conditions. In this case, as the velocity decreases, not only does the signal intensity increase, but also its shape changes, becoming an almost perfect Gaussian (for example, signal obtained at a flow velocity of $1.4 \mathrm{~mm} / \mathrm{s}$ ). This can be observed because as the flow velocity decreases, the contribution of radial diffusion, and thus of the Taylor regime, increases, allowing the plug to be compacted during its journey. That radial diffusion now has a greater weight can also be observed in figure $7 \mathrm{~b}$, as signals are no longer independent of the diffusion coefficient variations. Intuitively, with the same flow velocity of $2 \mathrm{~mm} / \mathrm{s}$, as D increases, the plug arrives at the receiver more compactly and less dispersed, showing up as the result of a common Taylorgram ${ }^{36}$. In the light of this, under the microfluidic conditions employed in this simulation, it can be studied how the Taylor regime, whenever the flow velocity decreases or the diffusion coefficient increases, affects the signal in a typical transport model for molecular communication. Here too, we have evaluated Intersymbol Interference in the same way as for mChannel conditions, setting a delay of 300 seconds between two consecutive bits. 
(a) $\quad-v=1.4 \mathrm{~mm} / \mathrm{s}-\mathrm{v}=2.3 \mathrm{~mm} / \mathrm{s} \quad-\mathrm{v}=5.9 \mathrm{~mm} / \mathrm{s}$
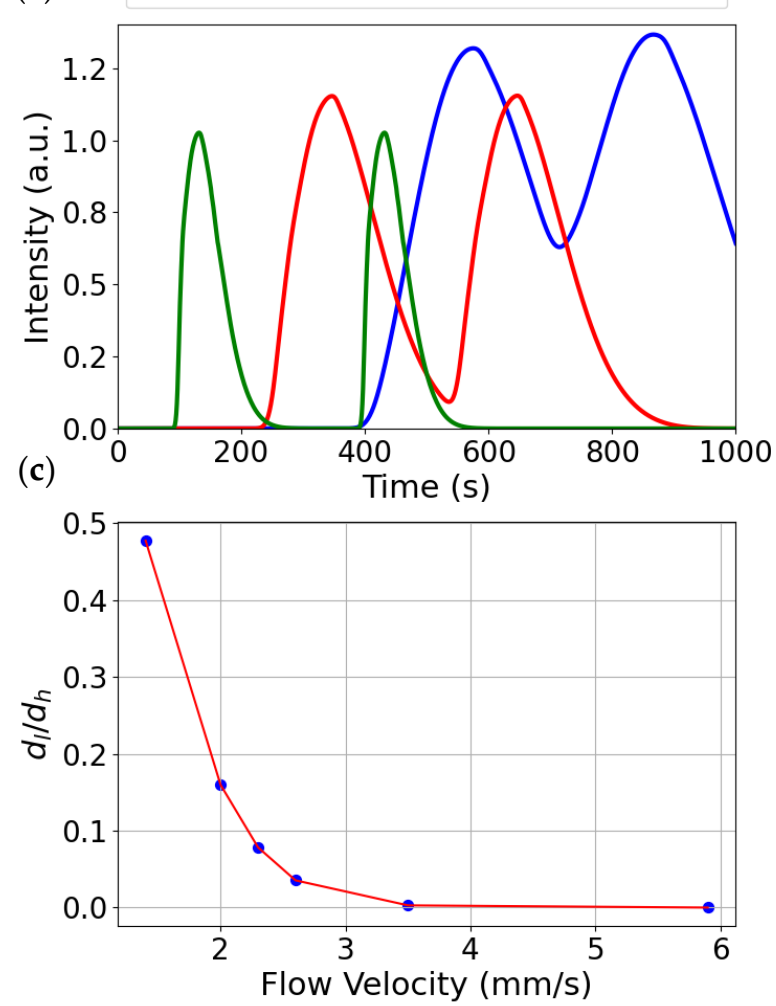
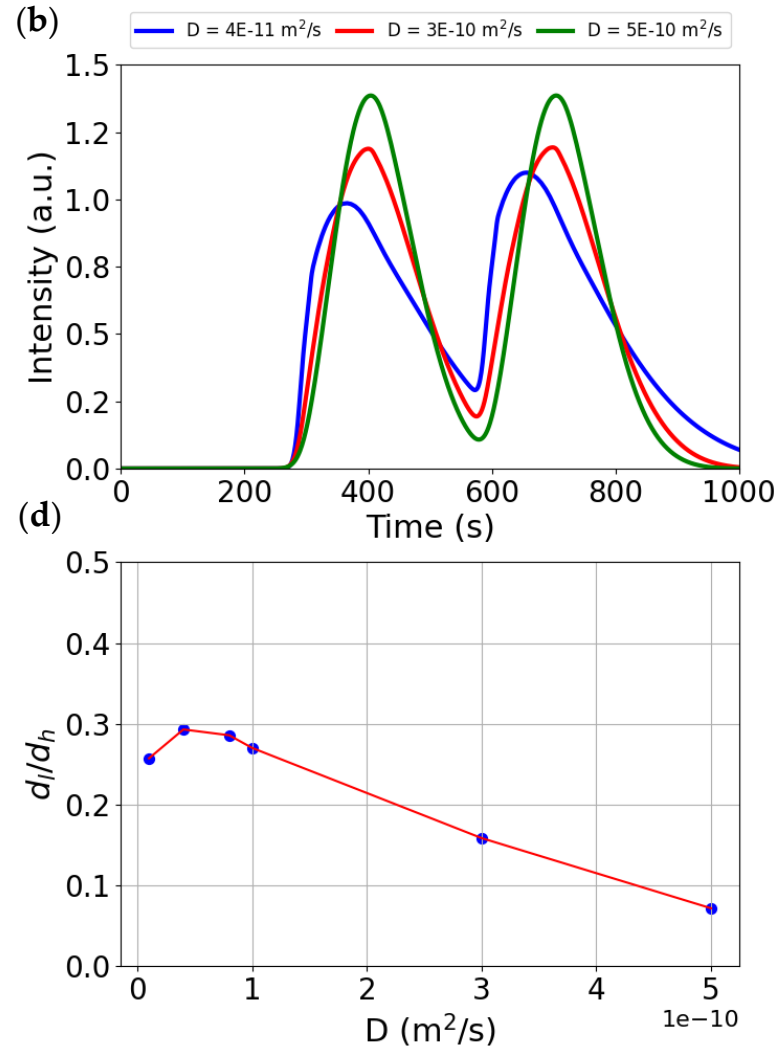

Figure 7. (a,b) Simulated signals representing two consecutive bits with a fixed delay between them of 300 seconds, colours are associated with different values of flow velocity and diffusion coefficient, respectively; (c,d) development of the dl/dh ratio as a function of flow velocity and diffusion coefficient, respectively.

Figure 8a shows that as flow velocity decreases ISI increase, as seen for mChannel simulations. Figure $8 \mathrm{~b}$ shows that ISI is also influenced by variations in the diffusion coefficient, as it can be observed that the $\mathrm{d}_{\mathrm{l}} / \mathrm{d}_{\mathrm{h}}$ ratio changes considerably within a range of $\mathrm{D}$ that differ by at most one order of magnitude. Obviously, with the above considerations and looking at figure $8 \mathrm{~d}$, the ISI effect decreases as the diffusion coefficient increases. We can call this condition in which Taylor diffusion clearly manifests itself due to the size of the channel in which transport has been simulated $\mu$ Channel condition.

In an overview, we can say that in what we have defined as mChannel conditions, if we wanted to send a bit sequence, to increase the information density and avoid reading errors due to ISI we would have to work only at higher flow rates. The performance does not change if we modify the diffusion coefficient of the transported substance, for example by varying the viscosity of the transport fluid or the size of the messengers (remember Stokes-Einstein's law). In any case it will not be possible to obtain compact Gaussian signals. Concerning $\mu$ Channel conditions, we can maintain a high bit rate even at lower flow velocities due to the Taylor effect that sets in as the flow velocities decreases. In this case the signals leave the asymmetrical distribution and become almost perfect Gaussians. In addition, an increase in the diffusion coefficient also makes it possible to observe a contribution from the Taylor regime that causes less plug dispersion. Thus, operate under $\mu$ Channel conditions allows us to have a wider range of flow velocities to obtain successive signals that are completely separated and to use smaller delays between signals without loss of information quality. 
In the light of these results, it was decided to set up a prototype for molecular communication under $\mu$ Channel conditions (see Materials and Methods) which, as has been seen, are those with which it is easiest to optimize the process of transporting messengers and thus information. To do this, four carbon dots suspensions with the same concentration but different viscosities were prepared, respectively of $1.0,2.5,5.0$, and $7.5 \mathrm{mPa} \cdot \mathrm{s}$ affecting the diffusion of information nanoparticles. The first one is a CQDs suspension in water only, while the others are suitable mixtures of water and glycerol. A carrier with the same viscosity was prepared for each CQDs suspension. Higher viscosities have not been considered because, operating under microfluidic conditions, the syringe system that allows continuous flow of the carrier does not maintain optimum performance.

Figure 9a shows the experimental signals obtained by varying the flow velocity

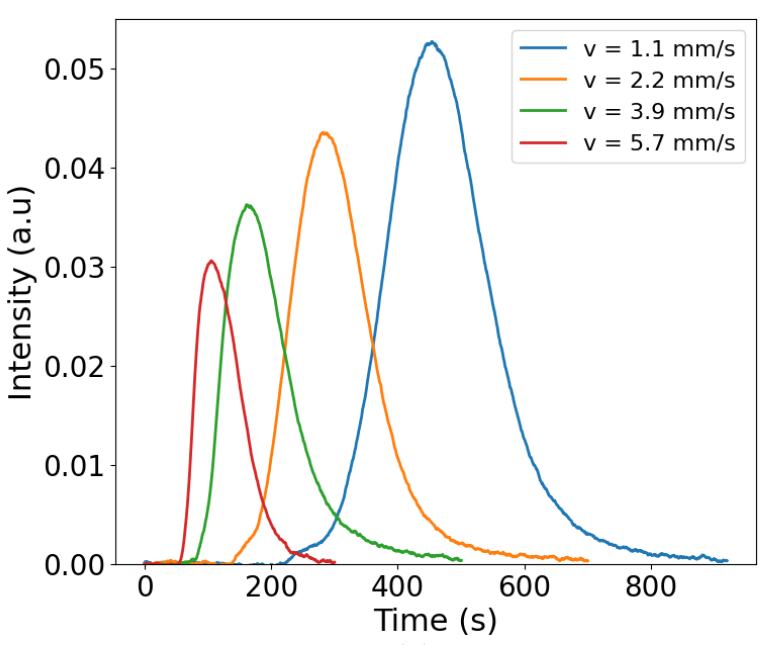

(a)

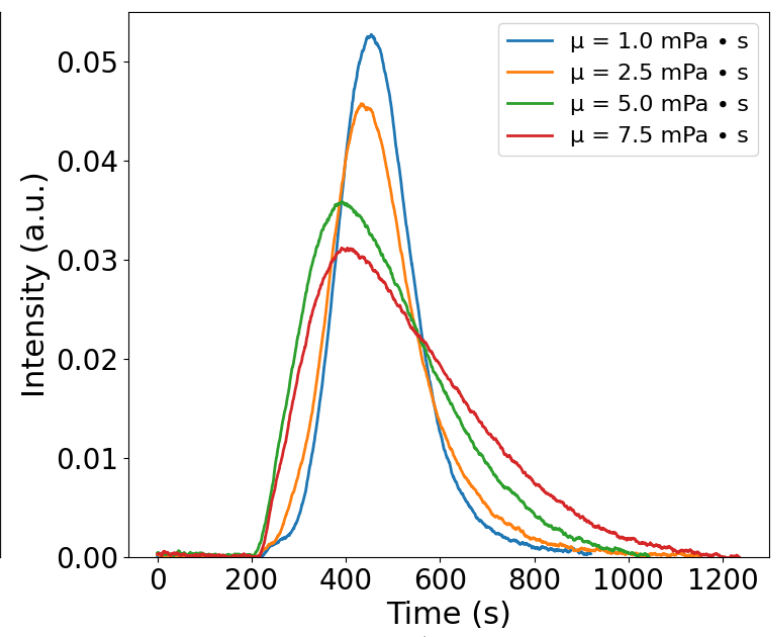

(b)

Figure 8. Experimental signals under microfluidic conditions; (a) colours are associated with different values of flow velocity using a CDs suspension water only $(1.0 \mathrm{mPa} \cdot \mathrm{s})$; (b) colours are associated with different values of viscosity, while a constant flow velocity of $1.1 \mathrm{~mm} / \mathrm{s}$ is used.

within a range similar to that which was used for the simulations. In this case, we operated with the suspension of CQDs in water, therefore at constant viscosity and diffusion coefficient. It is extremely clear that under experimental conditions the same trend of the signals seen in the simulations is obtained as the flow velocity decreases. In order to investigate the trend of the signals at constant flow velocity and varying diffusion coefficient, we obtained the signals represented in figure $9 \mathrm{~b}$ at varying viscosity of the entire communication channel. Considering carbon quantum dots that have average diameter of $10 \mathrm{~nm}^{32}$, the diffusion coefficient associated with each viscosity for the Stokes-Einstein equation is estimated to be $4.29 \mathrm{E}-11,1.72 \mathrm{E}-11,8.60 \mathrm{E}-12$, and $5.73 \mathrm{E}-12 \mathrm{~m}^{2} / \mathrm{s}$, respectively. Again, as with the theoretical results, as the viscosity decreases, hence as the diffusion coefficient increases, a larger Taylor effect is obtained resulting in a more intense and Gaussian signal. 

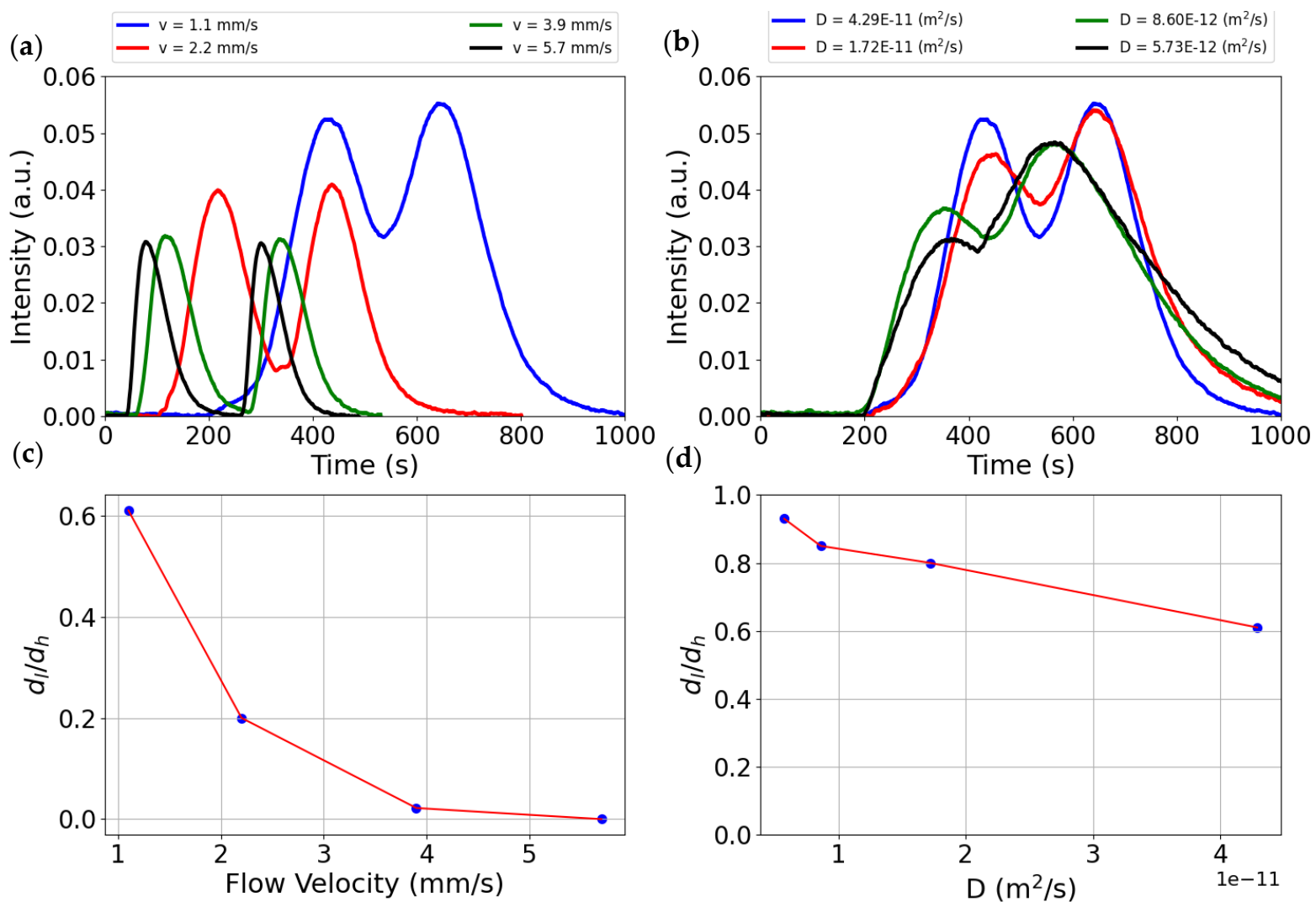

Figure 9. $(a, b)$ Experimental signals representing two consecutive bits with a fixed delay between them of 220 seconds, colours are associated with different values of flow velocity and diffusion coefficient, respectively; (c,d) development of the $\mathrm{d}_{\mathrm{l}} / \mathrm{d}_{\mathrm{h}}$ ratio as a function of flow velocity and diffusion coefficient, respectively.

Figure 10 confirms the Intersymbol Interference trend seen with the simulations as the flow velocity and diffusion coefficient vary in microfluidic conditions. For a simplified comparison, in the legend of graph $10 \mathrm{~b}$ and in the $\mathrm{x}$-axis of graph $10 \mathrm{~d}$ the values of the diffusion coefficients of the four different CQDs suspensions have been given instead of the viscosities. It is significant to observe the exponential trend in figure $10 \mathrm{c}$, which is also exactly reproduced in figure $8 \mathrm{c}$ by means of simulations: in both, completely separated signals are obtained at flow velocities of about $4 \mathrm{~mm} / \mathrm{s}$.

\section{Conclusions}

In this work, the comparison between $\mu$ Channel and mChannel conditions was studied in order to optimize the transport of information carriers for molecular communication. Through a theoretical study first, and an experimental campaign later, it was possible to analyze the benefits that the Taylor regime triggered in $\mu$ Channel - MoCo brings. While for mChannel - MoCo it has been seen that the only condition to ensure a good information density without incurring read errors is to operate at higher flow rates, in the case of $\mu$ Channel - MoCo, using the Taylor regime, optimization can be achieved by means of the flow rate and also the diffusion coefficient of the information particles. It is extremely encouraging to see the accuracy with which the experimental signals were obtained compared to the simulated ones. This allows us to confirm that in $\mu$ Channel - MoCo can be used a wider range of flow velocities to obtain successive signals that are completely separated and smaller delays between signals without loss of information quality. The study represents a solid foundation to the realization of new applications using the biological fluids of certain animals, such as insects, or the sap of plants.

Author Contributions: Conceptualization, N.T. and F.C.; methodology, F.C. and L.F.; software, F.C. and N.T..; validation, N.T; resources, N.T.; data curation, F.C.; writing-original draft 
preparation, F.C.; writing - review and editing, N.T.; visualization, F.C.; supervision, N.T.; project administration, N.T.; funding acquisition, N.T. All authors have read and agreed to the published version of the manuscript.

Funding: This research was funded by University of Catania, grant number Nati4Smart Piano di incentivi per la Ricerca 2020/2022 - Linea di intervento 2" and "The APC was funded by University of Catania".

Acknowledgments: Authors thank Dott. Giovanni Contarino for valuable effort in the laboratory.

Conflicts of Interest: The authors declare no conflict of interest.

\section{References}

1. Guo, W. et al. Molecular Physical Layer for 6G in Wave-Denied Environments. 33-39 (2021).

2. Ozdemir, F. \& Kargi, A. Electromagnetic Waves and Human Health. in Electromagnetic Waves (InTech, 2011). doi:10.5772/16343.

3. Guo, W., Mias, C., Farsad, N. \& Wu, J.-L. Molecular Versus Electromagnetic Wave Propagation Loss in Macro-Scale Environments. IEEE Trans. Mol. Biol. Multi-Scale Commun. 1, 18-25 (2015).

4. Nakano, T., Eckford, A. W. \& Haraguchi, T. Molecular communication. Mol. Commun. 9781107023, 1-179 (2011).

5. Nakano, T., Moore, M. J., Fang Wei, Vasilakos, A. V. \& Jianwei Shuai. Molecular Communication and Networking: Opportunities and Challenges. IEEE Trans. Nanobioscience 11, 135-148 (2012).

6. Atakan, B. \& Akan, O. B. An information theoretical approach for molecular communication. in 2007 2nd Bio-Inspired Models of Network, Information and Computing Systems 33-40 (IEEE, 2007). doi:10.1109/BIMNICS.2007.4610077.

7. Farsad, N., Yilmaz, H. B., Eckford, A., Chae, C.-B. \& Guo, W. A Comprehensive Survey of Recent Advancements in Molecular Communication. IEEE Commun. Surv. Tutorials 18, 1887-1919 (2016).

8. Martinez-Ricos, J., Agustin-Pavon, C., Lanuza, E. \& Martinez-Garcia, F. Intraspecific Communication Through Chemical Signals in Female Mice: Reinforcing Properties of Involatile Male Sexual Pheromones. Chem. Senses 32, 139-148 (2006).

9. Antunes, L. C. M. \& Ferreira, R. B. R. Intercellular communication in bacteria. Crit. Rev. Microbiol. 35, 69-80 (2009).

10. Farsad, N., Guo, W. \& Eckford, A. W. Tabletop Molecular Communication: Text Messages through Chemical Signals. PLoS One 8, e82935 (2013).

11. Khaloopour, L. et al. An Experimental Platform for Macro-Scale Fluidic Medium Molecular Communication. IEEE Trans. Mol. Biol. Multi-Scale Commun. 5, 163-175 (2019).

12. Bicen, A. O. \& Akyildiz, I. F. System-Theoretic Analysis and Least-Squares Design of Microfluidic Channels for Flow-Induced Molecular Communication. IEEE Trans. Signal Process. 61, 5000-5013 (2013).

13. De Luis, B., Llopis-Lorente, A., Sancenón, F. \& Martínez-Máñez, R. Engineering chemical communication between micro/nanosystems. Chem. Soc. Rev. 50, 8829-8856 (2021).

14. Nakano, T., Eckford, A. W. \& Haraguchi, T. Molecular Communication. (Cambridge University Press, 2013). doi:10.1017/CBO9781139149693.

15. Parcerisa Giné, L. \& Akyildiz, I. F. Molecular communication options for long range nanonetworks. Comput. Networks 53, 2753-2766 (2009).

16. Moritani, Y., Hiyama, S. \& Suda, T. Molecular communication for health care applications. in Fourth Annual IEEE International Conference on Pervasive Computing and Communications Workshops (PERCOMW'06) 5 pp. - 553 (IEEE, 2006). doi:10.1109/PERCOMW.2006.97.

17. Malak, D. \& Akan, O. B. Molecular communication nanonetworks inside human body. Nano Commun. Netw. 3, 19-35 (2012).

18. Fichera, L., Li-Destri, G. \& Tuccitto, N. Graphene Quantum Dots enable digital communication through biological fluids. Carbon N. Y. 182, 847-855 (2021).

19. Wang, Z. et al. Xylem- and Phloem-Based Transport of CuO Nanoparticles in Maize (Zea mays L.). Environ. Sci. Technol. 46, 4434-4441 (2012). 
20. White, P. J. Long-distance Transport in the Xylem and Phloem. in Marschner's Mineral Nutrition of Higher Plants 49-70 (Elsevier, 2012). doi:10.1016/B978-0-12-384905-2.00003-0.

21. Bray, D. SIGNALING COMPLEXES:Biophysical Constraints on Intracellular Communication. Annu. Rev. Biophys. Biomol. Struct. 27, 59-75 (1998).

22. Transport phenomena, R. B. Bird, W. E. Stewart, and E. N. Lightfoot, John Wiley and Sons, Inc., New York(1960). 780 pages.\$11.50. AIChE J. 7, 5J-6J (1961).

23. Taylor, G. \& A, P. R. S. L. Dispersion of soluble matter in solvent flowing slowly through a tube. Proc. R. Soc. London. Ser. A. Math. Phys. Sci. 219, 186-203 (1953).

24. Bello, M. S., Rezzonico, R. \& Righetti, P. G. Use of Taylor-Aris Dispersion for Measurement of a Solute Diffusion Coefficient in Thin Capillaries. Science (80-. ). 266, 773-776 (1994).

25. Mahfuz, M. U., Makrakis, D. \& Mouftah, H. T. Characterization of intersymbol interference in concentration-encoded unicast molecular communication. in 2011 24th Canadian Conference on Electrical and Computer Engineering(CCECE) 000164-000168 (IEEE, 2011). doi:10.1109/CCECE.2011.6030431.

26. Kilinc, D. \& Akan, O. B. Receiver Design for Molecular Communication. IEEE J. Sel. Areas Commun. 31, 705-714 (2013).

27. Llatser, I., Cabellos-Aparicio, A., Pierobon, M. \& Alarcon, E. Detection Techniques for Diffusion-based Molecular Communication. IEEE J. Sel. Areas Commun. 31, 726-734 (2013).

28. Tuccitto, N., Li-Destri, G., Messina, G. M. L. \& Marletta, G. Fluorescent Quantum Dots Make Feasible Long-Range Transmission of Molecular Bits. J. Phys. Chem. Lett. 8, 3861-3866 (2017).

29. Tuccitto, N., Li-Destri, G., Messina, G. M. L. \& Marletta, G. Reactive messengers for digital molecular communication with variable transmitter-receiver distance. Phys. Chem. Chem. Phys. 20, 30312-30320 (2018).

30. Fichera, L., Li-Destri, G. \& Tuccitto, N. Fluorescent nanoparticle-based Internet of things. Nanoscale 12, 9817-9823 (2020).

31. Luo, P. G. et al. Carbon “quantum” dots for optical bioimaging. J. Mater. Chem. B 1, 2116 (2013).

32. Tuccitto, N. et al. Carbon Quantum Dots as Fluorescence Nanochemosensors for Selective Detection of Amino Acids. ACS Appl. Nano Mater. 4, 6250-6256 (2021).

33. Kuran, M. S., Yilmaz, H. B., Tugcu, T. \& Akyildiz, I. F. Modulation Techniques for Communication via Diffusion in Nanonetworks. in 2011 IEEE International Conference on Communications (ICC) 1-5 (IEEE, 2011). doi:10.1109/icc.2011.5962989.

34. Mahfuz, M. U., Makrakis, D. \& Mouftah, H. T. Strength-based optimum signal detection in concentration-encoded pulsetransmitted OOK molecular communication with stochastic ligand-receptor binding. Simul. Model. Pract. Theory 42, 189-209 (2014).

35. Calì, F. et al. Carbon Quantum Dots from Lemon Waste Enable Communication among Biodevices. Chemosensors 9, 202 (2021).

36. Alizadeh, A., Nieto de Castro, C. A. \& Wakeham, W. A. The theory of the Taylor dispersion technique for liquid diffusivity measurements. Int. J. Thermophys. 1, 243-284 (1980). 